\title{
LA LITERATURA DEL CARIBE ANGLÓFONO: UNA REVISIÓN DE LA PRIMERA MITAD DEL SIGLO XX*
}

\author{
ANGLOPHONE CARIBBEAN LITERATURE: A REVISION \\ OF THE FIRST HALF OF THE CENTURY
}

Elsa Maxwell

Universidad de Chile. Santiago, Chile

elsamaxwell@gmail.com

Recibido: 27.03.2013. Aceptado: 10.10.2013.

Resumen: Basándose en investigaciones recientes que ponen en cuestión la idea de la inexistencia de literatura en el Caribe anglófono antes de "la generación del boom" de la década de 1950, este artículo busca contribuir al rescate y la difusión de escritores caribeños de la primera mitad del siglo. Presenta una revisión panorámica de los principales hitos de la producción literaria del Caribe anglófono entre 1900-1950, focalizando específicamente en el desarrollo del campo literario en Jamaica durante las dos primeras décadas del siglo, el surgimiento de las revistas literarias durante los '30 y' 40 y la importancia de la emigración a la metrópolis para escritores de estas generaciones.

Palabras clave: Poesía jamaiquina, revistas literarias antillanas, emigración.

Abstract: Based on recent research which calls into question the idea that literature did not exist in the Anglophone Caribbean before the 1950's "boom generation", this article contributes to the recovery and dissemination of first half of the $20^{\text {th }}$ Century Anglophone Caribbean writers. It presents a panoramic revision of the Anglophone Caribbean's literary production between 1900 and 1950, focusing specifically on the development of Jamaica's literary field during the first two decades of the century, the

* La autora quisiera agradecer a la Fundación Volcán Calbuco por apoyar esta investigación. 
rise of Caribbean little magazines in the $30 \mathrm{~s}$ and 40s, and the importance of emigration to the metropolis for writers from these generations.

Key words: Jamaican poetry, Caribbean little magazines, emigration.

\section{Introducción}

$\mathrm{E}$ L TEMA PRINCIPAL de este estudio es la producción literaria del Caribe anglófono de la primera mitad del siglo XX, periodo histórico que ha sido poco estudiado debido, en gran medida, al predominio de un paradigma crítico que niega la existencia de una literatura anglófona caribeña anterior a la "generación del boom" de la década de los cincuenta. En este sentido, busca aportar al proyecto recuperativo de escritores tempranos del Caribe anglófono, al presentar una revisión panorámica de los principales hitos de la producción literaria de esta región lingüística durante la primera mitad del siglo XX. Siguiendo la línea investigativa de Alison Donnell (2006) y Donnell y Lawson Welsh (1996), la primera parte de este trabajo aborda iniciativas literarias que surgen durante las dos primeras décadas del siglo, tomando como caso los esfuerzos por fomentar la producción, diseminación y lectura de escritura local en Jamaica. La segunda sección aborda las décadas de los 30 y 40 y destaca los esfuerzos por levantar revistas literarias en distintas partes de la región -Jamaica, Trinidad, y Barbados, entre otros países- poniendo particular énfasis en los desafíos enfrentados en términos culturales y monetarios que a menudo impedían la continuidad de ellas. La tercera sección aborda el patrón de emigración que comienza durante la primera mitad del siglo, debido en gran parte a los obstáculos enfrentados al intentar escribir en forma profesional durante este periodo de desarrollo cultural en la región.

En términos generales, el paradigma de la inexistencia de una literatura caribeña anglófona anterior a 1950 se fundamenta en la idea de que no había una escritura "auténtica" anterior a la publicación de los escritores caribeños asociados al "boom”, muchos de ellos autoexiliados en Inglaterra: George Lamming y Edward Kamau Brathwaite de Barbados, V.S. Naipaul de Trinidad, Wilson Harris, Sam Selvon y Edward Mittleholzer de la Guyana británica, y Roger Mais y V.S. Reid de Jamaica. Con el tiempo, 
estos autores serían canonizados como "la generación del boom" del Caribe anglófono y llegarían a constituir el punto de partida para el estudio del corpus de las Indias Occidentales (Donnell y Lawson Welsh, 1996).

La idea de que no había literatura en el Caribe antes de 1950, o que era imitativa y por ende inauténtica, se va forjando en el mismo contexto de la generación del boom, periodo en el cual aparecen numerosas referencias críticas al estado inexistente, muerto o dormido de la literatura caribeña durante la primera mitad del siglo ${ }^{1}$. Esta idea también se encuentra en los escritos de los mismos autores del boom autoexiliados en la metrópolis, cuyas reflexiones sobre la genealogía literaria de su generación a menudo apuntan a la inexistencia de modelos literarios caribeños anteriores y, por extensión, la ausencia de una tradición literaria caribeña antes de 1950. Muy ilustrativas de esta visión son las afirmaciones de C.L.R. James respecto a la literatura en Trinidad durante los treinta ("no había mucho que conocer”, 1996: 163) y los planteamientos de George Lamming en Los placeres del exilio (2007 [1960]), texto en el cual sostiene que la novela del Caribe anglófono emergió en la década del 50 en Londres y sin antecedentes antillanos previos:

El hecho histórico es que el "surgimiento" de aproximadamente una docena de novelistas en el Caribe británico con unos cincuenta libros en su haber y deshaber y todos publicados entre 1948 y 1958 constituye algo así como un fenómeno (54).

Como debe ser, el novelista fue el primero que relató la experiencia antillana desde dentro. Fue el primero en registrar la memoria antillana hasta donde le fue posible remontarse... Hemos visto antes nuestros ojos una actividad llamada escritura, que adopta la forma de novela, cristalizar sin tradición autóctona anterior de que tomar. Mittleholzer, Reid, Selvon y Roger Mais [...] son los primeros constructores de la que será una tradición en la literatura imaginativa antillana, una tradición que se dará por sentada o que servirá a los fines del análisis crítico que realicen antillanos de una generación anterior (68-69, cursiva mía).

1 "As late as 1966, A.J. Seymour declared that 'the 1920 s were a period when the British Caribbean was still literarily asleep', and in 1970, despite paying it considerable attention, the pre-1930 period was branded by Kenneth Ramchand as 'life without fiction"” (Donnell y Lawson Welsh, 1996: 13). 
Por cierto Lamming se está refiriendo específicamente a la emergencia del género de la narrativa caribeña, aunque su descripción de los novelistas del boom como los pioneros de la primera tradición de escritura imaginativa caribeña implícitamente sugiere la inexistencia de la escritura creativa antes del boom.

Debido a que Lamming y otros integrantes del boom llegarían a ejercer un papel influyente en la fijación de los estándares críticos empleados durante las décadas del 70 y 8o, la idea de la inexistencia de una tradición literaria caribeña anglófona anterior a 1950 también tomaría fuerza en el plano de la crítica contemporánea, donde frecuentemente se refiere al boom como la primera generación de escritores del Caribe anglófono. Asimismo se ve reflejado en el posicionamiento de la cronología literaria caribeña a partir de 1950 en adelante, lo cual ha producido estudios e historias literarias que suelen dejar de lado las generaciones anteriores de poetas, novelistas y ensayistas que activamente buscaban fomentar la producción literaria en la región. Una de las pocas excepciones se encuentra en las frecuentes referencias a C.L.R. James, cuya obra temprana publicada en Londres ${ }^{2}$ durante la década del 30 es a menudo estudiada en relación a la generación del boom, pero no en relación al periodo literario en el que fue escrita y publicada ${ }^{3}$.

Pero más allá de la atención crítica dirigida a la obra de James, sigue siendo difícil encontrar estudios enfocados específicamente en el periodo literario anterior a 1950, tal como señala Donnell (2006) en su reciente libro sobre la literatura del Caribe anglófono antes del boom. Sin embargo,

${ }^{2}$ James publica dos libros en la metrópolis durante la década del 30: Minty alley (1936), la primera novela caribeña publicada en Londres, y The black jacobins (1938), la primera historia de la revolución haitiana escrita por un antillano.

${ }^{3}$ Considérese, por ejemplo, la cronología propuesta por Simon Gikandi (1992) en la cual James aparece junto a dos de los escritores más emblemáticos del boom anglófono: "The most important literary and cultural documents in the Caribbean tradition -Aimé Césaire's Cahier, Frantz Fanon's Black skin, white masks, C.L.R. James's The black jacobins, V.S. Naipaul's A house for Mr. Biswas, and George Lamming's In the castle of my skin - were produced in exile. Because of this simple fact, any attempt to map the direction in which contemporary Caribbean writing has developed, or to account for the emergence of a distinctly Caribbean literary tradition, must investigate the phenomenon of exile as a historical and existential condition. In other words, exile and the displacement it engenders constitute the ground zero of West Indian Literature, its radical point of departure..." (33). 
investigaciones recientes como la antología de Donnell y Lawson Welsh (1996), han demostrado que efectivamente existieron importantes iniciativas literarias durante la primera mitad del siglo que, si bien no logaron el éxito internacional de la generación del boom, constituyen un aporte fundamental al desarrollo de las letras en el Caribe anglófono.

\section{Hacia una literatura local: el caso de Jamaica entre 1900-1920}

Desde principios del siglo surgen múltiples iniciativas literarias en el Caribe que, de acuerdo al análisis de Gail Low (2010), constituyen un importante espacio de producción cultural local frente al monopolio metropolitano que controlaba la mayor parte de la publicación y distribución de libros y otros textos culturales. El impulso literario local es evidente en la fundación de varias revistas literarias y proyectos editoriales en las Indias Occidentales durante las primeras décadas del siglo, así como en el compromiso de importantes diarios caribeños con la publicación de autores locales en un formato económico y accesible. En primera instancia, estas iniciativas tendían a provenir de la clase criolla adinerada que estaba dispuesta, en algunos casos, a invertir recursos propios a fin de promover el desarrollo cultural de las colonias inglesas mediante la diseminación de lo que se consideraba como alta cultura. En este sentido, una parte importante de la producción literaria de este periodo se caracteriza por su lealtad e identificación con la metrópolis, evidente, por ejemplo, en la propuesta estética de J.E.C. McFarlane, el fundador de la rama jamaiquina del Empire Poetry League, y en poemas como los de Albinia Hutton que rinden homenaje a la madre patria británica. Es por esta misma razón que Donnell (2006) sugiere que los autores del boom -muchos de ellos comprometidos con la causa anticolonial- negaron tener un parentesco literario con las obras provenientes de este periodo, puesto que muchas de ellas se caracterizaban por su insistente adherencia estética y política al Imperio británico. En este mismo periodo, sin embargo, es posible detectar un creciente interés en la afirmación de una identidad local distinta a la de la metrópolis, visible en el proyecto literario de Tom Redcam, quien fundó el All Jamaican Library en 1904 para incentivar la escritura por y para jamaiquinos, así como en los poemas de Claude McKay, cuya redacción de versos en el 
patois jamaiquino marca una ruptura importante con los patrones poéticos dominantes que no consideraban el creole como una lengua idónea para la expresión literaria. En este contexto, el estudio que se presenta a continuación busca dar cuenta de las iniciativas de las múltiples corrientes literarias y estéticas que coexistían durante este periodo, tomando en consideración que a menudo se entrelazaban entre sí.

Conviene comenzar señalando un conjunto de proyectos literarios que emergen en Jamaica durante las primeras décadas del siglo, entre ellos, el ya señalado All Jamaican Library de Tom Redcam (seudónimo de Thomas Henry MacDermot), establecido para "promover lo distintivo de la identidad y literatura jamaiquina" mediante la publicación de textos locales que reflejaran "las preocupaciones de la isla" (citado en Low, 2010: 208, traducción mía). Por ello mismo, se trataba de una de las tempranas iniciativas enfocadas en el desarrollo de una literatura jamaiquina distinta de la inglesa, pues aunque MacDermot profesaba su lealtad a la corona, buscaba al mismo tiempo incentivar la producción de una escritura autóctona que reflejara la realidad jamaiquina. En términos materiales, MacDermot empleó las instalaciones del periódico que editaba en ese tiempo - The Jamaican Times- para publicar cuatro libros entre 1904 y 1909, fijando su precio en un shilling para facilitar e impulsar la lectura y la venta de éstos (Low, 2010). Dos de los cuatro libros incluían poemas del mismo MacDermot, quien, al igual que otros escritores criollos, empleaba la editorial que había creado para publicar sus propias obras también. Una iniciativa parecida se encuentra en el proyecto editorial de Herbert de Lisser, un joven escritor previamente publicado por MacDermot que a los 26 años llegó a ocupar el cargo de editor de The Gleaner, uno de los diarios más importantes de Jamaica. Bajo su dirección hasta 1944, The Gleaner publicó las obras poéticas y narrativas de diversas tendencias, entre ellas: la novela con que De Lisser inauguró su carrera novelística, Jane’s career (1914), notable por ser una de las primeras narrativas jamaiquinas protagonizada por una campesina negra; los primeros poemarios de la poeta negra Una Marson (Tropic reveries y Heights and depths, 1930 y 1931, respectivamente), quien más adelante desempeñaría un papel importante en el movimiento feminista y nacionalista en Jamaica; y la antología poética Songs of empire (1932), una colección de poemas editada por Constance Hollar que se unían en torno a su fidelidad hacia la Corona británica y el proyecto imperial (Donnell y 
Lawson Welsh, 1996). Según Low (2010), De Lisser a menudo vendía los libros a cincuenta por ciento del costo de producción a fin de fomentar su venta entre la población, una filosofía reflejada además en la fundación de Planter's Punch, una revista relativamente económica dedicada a la publicación de autores locales, siendo al mismo tiempo el medio por el cual De Lisser publicó sus restantes nueve novelas.

Quien ejerció un papel igualmente influyente en el desarrollo de las letras jamaiquinas fue el poeta J.E.C. McFarlane, fundador del Jamaican Poetry League (1923), una rama del Empire Poetry League que desempeñó un rol crucial en la visibilización y diseminación de la poesía local mediante la organización de tertulias, conferencias, publicaciones anuales y premios. Para muchos jóvenes poetas de la época, el Poetry League constituía un importante foro para el reconocimiento de su trabajo, dando aliento a una nueva generación de poetas, entre ellos Claude McKay y Una Marson. Al mismo tiempo, no obstante, McFarlane formaba parte de una de las corrientes literarias más tradicionales del periodo, por lo que tendía a publicar obras que reflejaban la lealtad hacia la Corona británica y los estándares literarios establecidos en la metrópolis. Por este motivo, McFarlane veía la producción literaria asociada al Jamaican Poetry League como una extensión de la tradición literaria británica, reflejado, por ejemplo, en el discurso de apertura que dio en 1935: "como representantes de una gran tradición les ofrecemos Poesía, sobre la cual [...] descansa la verdadera fundación del Imperio" (1996a: 175, traducción mía). La identificación de McFarlane con el Imperio asimismo se ve reflejada en su concepción de la misión de la poesía, basada en la idea de que ésta no debería representar la realidad sino trascenderla, con el fin de elevar el espíritu del lector más allá de su existencia material. De este modo, la poesía constituía un indispensable mecanismo para levantar el estado moral y cultural de los pobres, aunque no era misión del poeta encargarse del problema de las precarias condiciones materiales en que vivía la mayor parte de la población jamaiquina (1996a).

Indudablemente, la concepción de la poesía como una expresión desconectada de la realidad social sería fuertemente cuestionada, y hasta descartada, por las generaciones que surgen en la década del 50, que en su mayoría estaban comprometidas con el anticolonialismo político y cultural. Pero es interesante notar que mucho antes del boom ya habían aparecido distin- 
tas voces poéticas que estaban experimentando con el uso de la poesía para reflexionar sobre la realidad social de la isla, sobre todo en relación a las divisiones y desigualdades entre las razas. En esta línea, cabe señalar el poema de Claude McKay, "My native land my home" publicado en su primer poemario Songs of Jamaica en 1912. Presentado en un formato aparentemente tradicional (estrofas rimadas de cuatro líneas), se trata de un poema plasmado de un sentido de pertenencia nacional que sutilmente invierte las relaciones coloniales al posicionar a Jamaica como la madre tierra, al mismo tiempo que reivindica la pertenencia y presencia negra en la isla frente a la dominación buccra (blanca): "Jamaica is de nigger's place, / No mind whe' some declare; / Although dem call we 'no-land race', / I know we home is here... / You give me life an' nourishment, / No udder land I know / My lub I neber can repent, / for all to you I owe..." (McKay, 1996: 64). Como evidencian los versos citados, McKay realiza una innovación lingüística importante al reemplazar el inglés estándar por la lengua popular jamaiquina -el patois- en ese periodo aún considerada inadecuada para la expresión literaria ${ }^{4}$. En este sentido, el poema de McKay genera una ruptura significativa con los patrones literarios de la época, no sólo en términos de contenido (los conflictos raciales) sino también en términos formales (el empleo del creole). Una Marson ofrecería una propuesta similar en la poesía que escribió a raíz de su primera residencia en Inglaterra (1932-1937) y el racismo que enfrentó allí. En 1933 escribió el poema "Nigger", verso que aborda abiertamente el tema de la discriminación racial: "The called me 'Nigger', / Those little white urchins, / They laughed and shouted / As I passed along the Street, / They flung it at me: / 'Nigger! Nigger! Nigger!" (Marson, 1996c: 140). El tercer poemario de Marson, The moth and the

${ }^{4}$ Considere, por ejemplo, el juicio de McFarlane respecto del uso del creole en la poesía de McKay: "Like [Robert] Burns, he [McKay] expresses the soul of his people in a medium created by his people... But as characteristic and valuable as are these dialect poems they do not embrace, any more than did those of Burns, the full range of McKay's poetic powers. Dialect, as I have pointed out elsewhere, is a 'broken tongue' with which it is impossible to build an edifice of verse possessing the perfect symmetry of finished art" (1996b). La clasificación del creole como una lengua rota sería refutada más adelante por autores como Edward Kamau Brathwaite, cuya afirmación del uso del "nation language" cobraría vigencia en el contexto de la independencia y los concomitantes procesos de construcción nacional. 
star (Kingston, 1937), también refleja su enfrentamiento con el racismo en la metrópolis, pero a diferencia de su poesía anterior, emplea el patois, indicativo indudablemente de su creciente adherencia a la causa anticolonial y nacionalista. Incluye poemas como "Quashie comes to London", verso que reconfigura la mirada colonial al describir Londres desde la perspectiva de un inmigrante antillano negro, y "Kinky hair blues", un poema sobre la concepción metropolitana de la belleza femenina en relación a la mujer negra: "Gwine find a beauty shop / Cause I ain't a belle... / The boys pass me by, / The say I's not swell... / I hate dat ironed hair / And dat bleaching skin... / But I'll be all alone / If I don’t fall in” (Marson, 1996b: 137).

\section{0-1940: El auge de las revistas literarias}

Como muestra la producción poética tardía de Una Marson, las décadas de los 30 y 40 se caracterizan por la emergencia de nuevos actores y voces críticas que buscaron traer a la luz pública el malestar social y económico en que vivía la mayor parte de la población caribeña. Desde los 30 en adelante, trabajadores y algunos sectores de la clase media gatillados por el empeoramiento de las condiciones socio-económicas producto de la Gran Depresión, comenzaron a organizarse frente a la autoridad colonial, llevando a cabo una serie de huelgas laborales y manifestaciones masivas en Trinidad y Tobago, Barbados, la Guyana Inglesa y Jamaica, demandando, entre otras cosas, mayor representación y participación en el manejo de los gobiernos locales. De estas movilizaciones nacieron los primeros sindicatos y partidos políticos que más adelante serían claves en los procesos de descolonización de la región. En este contexto, es interesante notar la simultánea fundación de varias revistas culturales y literarias de carácter crítico, entre ellas The Cosmopolitan, Trinidad y The Beacon, que al igual que las nuevas organizaciones políticas, buscaron poner en cuestión el status quo reinante, ya sea en términos culturales, económicos o de género. Estas revistas representan un significativo impulso político-cultural en la región, pese al hecho de que la mayor parte de ellas no logró sobrevivir más de un par de años debido a las precarias condiciones económicas y, en algunos casos, debido a la presión ejercida por los sectores más conservadores de las sociedades coloniales. Las revistas que nacen en la década de los 40, en 
cambio, son beneficiadas por un mejor clima económico, factor indudablemente decisivo en su relativa longevidad.

Respecto a las revistas fundadas en el contexto de la Depresión, cabe señalar a The Cosmopolitan, establecida por Una Marson en 1928 (cuando tenía sólo veinticuatro años) y financiada por un empresario ligado a los bienes raíces. Con la revista, Marson buscaba fomentar la producción literaria en particular y las artes en general, pero al mismo tiempo se trataba de una revista dirigida especialmente a las mujeres, declarando osadamente que "Esta es la era de la mujer: lo que ha hecho el hombre lo puede hacer la mujer" (citado en Jarrett-Macauley, 1998: 30, traducción mía). Como afirma Jarrett-Macauley, biógrafa de la poeta, The Cosmopolitan:

se trataba del empoderamiento de las mujeres por sí mismas. Una [Marson] buscaba rescatar de la fría desgracia nacional a la secretaria de pocos recursos, a la vendedora ambulante asidua, a la madre soltera y a la mujer cesante. Ella publicaba [...] informes de conferencias de mujeres, y encontró una forma, mucho antes de la existencia del movimiento jamaiquino de mujeres, para expresar los ideales feministas que hoy en día reconocemos (1998: 30, traducción mía).

Pionera en su medio, The Cosmopolitan constituye una propuesta significativa en términos de los derechos de las mujeres y los trabajadores, siendo además una de las primeras revistas de su tipo en el Caribe. No obstante (o quizás debido a ello) sólo logró sobrevivir tres años, recibiendo un duro golpe financiero tras la caída de la bolsa en Wall Street y la posterior retirada de varias agencias publicitarias que la habían sustentado. Por un tiempo, Marson se asoció con una co-editora a fin de mantener a flote la iniciativa, pero las inoportunas condiciones económicas, junto con las presiones por modificar el contenido y el enfoque de la revista, resultaron en el cierre de la publicación en 1931 (Jarrett-Macauley, 1998).

Una experiencia similar se encuentra en las revistas asociadas al Beacon Group en Trinidad y Tobago a principios de los años 30. Fundada en 1929, la revista Trinidad fue el producto de la colaboración entre Alfred Mendes, C.L.R. James y un grupo de jóvenes unidos en torno a su interés en la cultura y el pensamiento crítico. En gran medida, el grupo representaba una ruptura con el conservadurismo cultural de las clases medias y altas de Trinidad, reclamando "la hipocresía, el obscurantismo y la claustrofobia 
general de la sociedad trinitense" (Gomes, 1996: 166, traducción mía) aún bajo la influencia de los estándares victorianos. En este contexto, la revista Trinidad sirvió como una importante plataforma para James, siendo el medio donde publicó algunos de sus primeros cuentos cortos, entre ellos "Triumph", que narra un altercado entre tres mujeres de los barrack yards (poblaciones) sujetas a distintos niveles de opresión racial y patriarcal. Además de estimular la carrera temprana de James, las tertulias y publicaciones de este grupo proporcionaron un impulso importante a la carrera novelística de dos otros escritores de Trinidad: Alfred Mendes (Pitch Lake, 1934 y Black Fauns, 1935) y R.A.C. DeBoissiere (Crown Jewel, 1952 y Rum and Coca Cola, 1956) (Marquez, 1989).

En 1931, Trinidad sería seguida por The Beacon, una revista fundada, editada y publicada por Albert Gomes, y financiada en gran parte por su padre. Según cuenta Gomes en sus memorias, The Beacon era uno de los pocos espacios de encuentro para una nueva generación de jóvenes ansiosos por alejarse de las mentalidades anticuadas de la cultura dominante. Por ello Gomes sostiene que The Beacon implicó mucho más que una revista literaria, incluyendo no sólo poesía y cuentos sino también ensayos sobre temas de trascendencia nacional, entre ellos la ley de divorcio de 1932, convirtiéndose así en un importante foro de debate para un conjunto de hombres jóvenes disconformes con el status quo (1996). Dada la perspectiva crítica, los artículos que aparecían en The Beacon rápidamente llamaron la atención de las autoridades, preocupadas por la creciente popularidad de una revista que estaba comprometida con la crítica social y política. Por ejemplo, cuando Gomes escribió un artículo titulado "Black Man" sobre la discriminación racial en el sur de los Estados Unidos, llegaron tres policías a su casa para revisar sus papeles e interrogarle sobre el origen del material que había usado. La publicación de artículos que criticaban el papel de la Iglesia también generó la recriminación de dicha institución, la cual mantuvo un fuerte lobby financiero y discursivo contra la revista. Cuando el padre de Gomes finalmente decidió no seguir financiando la imprenta de la revista -en algunas ocasiones había pagado la totalidad de la impresión cuando su hijo no contaba con auspiciadores- la revista quedó descontinuada en 1933.

La década de los 40, en cambio, se caracteriza por la fundación de numerosas revistas literarias de carácter nacional y regional que se destacan 
por sus largas trayectorias: Bim (Barbados), por ejemplo, fue fundada por Frank Collymore en 1942 y estuvo en circulación por más de cincuenta años, mientras que Kyk-Over-Al (Guyana Inglesa) y Focus (Jamaica) fueron publicadas entre 1945-1961 y 1943-1960, respectivamente. En los últimos años mucho ha sido escrito sobre el importante papel de estas revistas en el cultivo de la literatura caribeña, particularmente en relación al boom ${ }^{5}$, y por eso sólo quisiera hacer unos breves comentarios que permiten reflexionar sobre su relativo éxito en relación a las dificultades experimentadas por las revistas fundadas durante los 30 . Como se ha señalado, y como podrá percatarse el lector, las condiciones económicas de este periodo fueron notoriamente mejores que las de la década anterior, por lo que se puede suponer que hubo mayores recursos para la inversión en iniciativas culturales, generando circunstancias más estables para su publicación y circulación. Por otra parte, los editores de las revistas fundadas en los 40 parecen haber tenido posicionamientos políticos menos radicales y, por ello mismo, redes sociales más amplias que aseguraron una mayor circulación de las revistas. Por ejemplo, el influyente editor de Bim, Frank Collymore, fue un profesor del prestigioso colegio Combermere School conocido por su conservadurismo político pese a su firme compromiso con la publicación de autores de otras tendencias políticas, tales como Lamming y Brathwaite. El editor de Kyk-over-al, A.J. Seymour, también provenía de la elite educada, realizando sus estudios en el prestigioso Queen's College, mientras que Edna Manley, editora de Focus, era la esposa de Norman Manley, uno de los políticos más importantes durante el periodo de la independencia jamaiquina. Falta mayor investigación sobre este asunto, pero a primera vista da la impresión de que las revistas fundadas durante la década de los 40 contaron no sólo con mejores condiciones económicas, sino también con mejores redes de apoyo en términos políticos y culturales, factores que indudablemente fueron claves en su continuidad. (2010).

${ }^{5}$ Ver Donnell (2006), Donnell y Lawson Welsh (1996), Low (2010) y Baugh 


\section{"Why I love, and leave, Jamaica": La conflictiva decisión de emigrar}

No obstante los múltiples esfuerzos para fomentar la producción literaria local durante la primera mitad del siglo XX, seguía siendo arduo para los escritores proyectarse como profesionales. Pocas revistas literarias tenían los recursos para pagar a sus colaboradores y seguía siendo muy difícil publicar novelas en la región por la falta de casas editoriales estables y un público lector más amplio. Por ello, muchos escritores de la primera mitad del siglo -anticipándose a la generación del boom- optaron por publicar en Londres o los Estados Unidos y, en la medida de lo posible, emigrar a dichos lugares para acceder con mayor facilidad a las redes literarias y las casas editoriales. Ya en 1912 Claude McKay había decidido emigrar a los Estados Unidos tras la publicación de sus dos primeros poemarios en Kingston, empleando el dinero que había ganado de un premio del Jamaican Institute of Arts and Sciences para costear su pasaje. Pasó sus primeros dos años estudiando en Kansas State College y luego se trasladó a Nueva York, donde se convertiría en uno de los impulsores más destacados del llamado Renacimiento negro en Harlem, lugar donde publicaría una parte importante de su obra (Gates y McKay, 2004).

C.L.R. James también optó por emigrar, radicándose en Inglaterra en 1932 a instancias de su amigo Learie Constantine, un jugador de cricket que le ayudó a conseguir trabajo. En la metrópolis, James comenzó una larga carrera literaria y periodística, partiendo con la publicación de su novela Minty alley, escrita en 1929 en Trinidad pero no publicada hasta 1936 en Londres, y luego su celebrada historia de la revolución haitiana, The black jacobins en 1938. Como señala James (1996), para muchos jóvenes negros, la única forma de desarrollar una carrera literaria e intelectual era emigrar a la metrópolis, a diferencia de sus compañeros no-negros del grupo -Gomes, Mendes, de Boissiere, entre otros- cuyo estatus socio-económico y racial les permitía seguir desarrollándose en Trinidad o viajar a los Estados Unidos donde podían perfeccionar su escritura: "Nosotros [los negros] fuimos en una dirección; los muchachos blancos fueron en otra. Éramos negros y la única manera de hacer lo que nos interesaba implicaba viajar al extranjero" (164, traducción mía). 
Al igual que James, Marson viajó a Londres por primera vez en 1932, iniciando así una serie de residencias largas en la capital inglesa. Allí Marson ejerció un papel clave en la fundación de Caribbean Voices, un programa radial de la BBC que más adelante sería uno de los principales promotores de nuevos autores antillanos durante el periodo del boom. En este sentido, Marson siempre se mantuvo conectada con el ámbito literario jamaiquino, escribiendo en 1949 un breve pero importante ensayo titulado "We want books - But do we encourage our writers?", denunciando la imposibilidad de proyectarse como escritor en la isla, lamentando que ahí sólo había una docena de escritores profesionales que lograban vivir de su escritura (1996a). Para Marson, el problema no sólo radicaba en la falta de casas editoriales sino en la poca valoración, el desinterés, e incluso la deshonra asociada a la escritura en Jamaica:

Me dicen y yo sé que en Jamaica el escritor joven es visto como alguien extraño. Si es un funcionario público, sus superiores piensan que sería un mejor funcionario si no estuviera interesado en la literatura y si no tuviera ambiciones literarias... En su casa, esconde sus poemas o ensayos por el miedo a que se rían de él... No creo que el público [jamaiquino] pudiera identificar a nuestros escritores principales, tampoco les interesa (1996a: 185-186, traducción mía).

Marson concluye la cita notando, con un dejo de ironía, que incluso, un taxista o un empleado del aseo en Londres podrían convertirse en novelistas debido a las numerosas oportunidades literarias y casas editoriales.

Para cerrar, quisiera señalar unas líneas de un ensayo del prolífico novelista, cuentista y poeta jamaiquino Roger Mais, "Why I love, and leave, Jamaica" (1950), texto que puede ser leído como un puente entre los escritores de la primera mitad del siglo y los de la segunda, en cuanto expresa una experiencia común entre ellos: la desvalorización del escritor creativo en las sociedades caribeñas coloniales. Escrito en 1950 -el año que simbólicamente marca el inicio del boom- "Why I love, and leave, Jamaica" retoma algunas de las críticas hechas por Marson, al mismo tiempo que anticipa las afirmaciones de Lamming y Brathwaite sobre el carácter filisteo de las clases educadas caribeñas del periodo: 
No pienso tener remordimientos al dejar atrás Jamaica... los valores que he adoptado para mí mismo no encajan aquí, no importan, no son conocidos, salvo en un círculo tan reducido que es casi incestuoso... Hay en este país, desgraciadamente, una torre atrincherada de la mediocridad, cercana e inexpugnable... que ha adquirido un cuerpo de opiniones tan mediocres que es inútil intentar hacer una abolladura en su engreimiento, su exclusividad y su indiferencia hacia cualquier cosa que no cabe cabalmente dentro de su limitado alcance e influencia... Los filisteos están acampados aquí (2-4, traducción mía).

\section{Referencias}

Baugh, E. (2010). The blossoming of Caribbean literature: The life and work of Frank Collymore. The Walter Rodney Lecture, University of Warwick, 18 March 2010. Recuperado de http://www2.warwick.ac.uk/fac/arts/ccs/ eventsnew/walterrodneylecture/rodney2010/walter_rodney_lecture_ march_2010.pdf

Donnell, A. y Lawson Welsh, S. (eds.). (1996). The routledge reader in Caribbean literature. London: Routledge.

Donnell, A. (2006). Twentieth-century Caribbean literature: Critical moments in Anglophone literary history. London: Routledge.

Gates, Jr. H. L. y McKay, N. Y. (eds.). (2004). The Norton anthology of African American literature. $2^{\mathrm{a}}$ edic. New York: W.W. Norton.

Gikandi, S. (1992). Writing in Limbo: Modernism and Caribbean literature. Ithaca: Cornell University Press.

Gomes, A. (1996). Through a maze of colour. En Donnell, A. y Lawson Welsh, S. (eds.), The Routledge reader in Caribbean literature (pp. 166-171). $2^{\mathrm{a}}$ edic. London: Routledge.

James, C.L.R. (1996). Discovering literature in Trinidad: The nineteen thirties. En Donnell, A. y Lawson Welsh, S. (eds.), The Routledge reader in Caribbean literature (pp. 163-165). $2^{\text {a }}$ edic. London: Routledge.

Jarrett-Macauley, D. (1998). The life of Una Marson, 1905-65. Manchester: Manchester University Press.

Lamming, G. (2007 [1960]). Los placeres del exilio (trad. María Teresa Ortega Sastrique). La Habana: Fondo Editorial Casa de las Américas.

Low, G. (2010). Publishing histories. En Shirley Chew y David Richards (eds.), A concise companion to postcolonial literature (pp. 204-229). Oxford: Wiley-Blackwell. 
Mais, R. (1950). Why I love, and leave, Jamaica. En UWI Mona Library Digital Collection (pp. 1-5). Recuperado de http://library-contentdm.mona.uwi. $\mathrm{edu} / \mathrm{u}$ ?/RMstories, 1624

Marquez, R. (1989). Nationalism, nation, and ideology. En Knight, F. W. y Palmer, C. A. (eds.), The modern Caribbean (pp. 293-340). Chapel Hill: The University of North Carolina Press.

Marson, U. (1996a). We want books - But do we encourage our writers? En Donnell, A. y Lawson Welsh, S. (eds.), The Routledge reader in Caribbean literature (pp. 185-186). London: Routledge, $2^{\text {a }}$ edic.

(1996b). Kinky hair blues. En Donnell, A. y Lawson Welsh, S. (eds.), The Routledge reader in Caribbean literature (pp. 137-138). $2^{\text {a }}$ edic. London: Routledge.

(1996c). Nigger. En Donnell, A. y Lawson Welsh, S. (eds.), The Routledge reader in Caribbean literature (pp. 140-142). London: Routledge, $2^{\text {a }}$ edic.

McFarlane, J.E.C. (1996a). The challenge of our time. En Alison Donnell y Sarah Lawson Welsh (eds.), The Routledge reader in Caribbean literature (pp. 174-176). $2^{\text {a }}$ edic. London: Routledge.

(1996b). Claude McKay. En Donnell, A. y Lawson Welsh, S. (eds.), The Routledge reader in Caribbean literature (pp. 97-98). $2^{\mathrm{a}}$ edic. London: Routledge.

McKay, Claude. (1996). My native land my home. En Alison Donnell y Sarah Lawson Welsh (eds.), The Routledge reader in Caribbean literature (pp. 64-65). London: Routledge. 